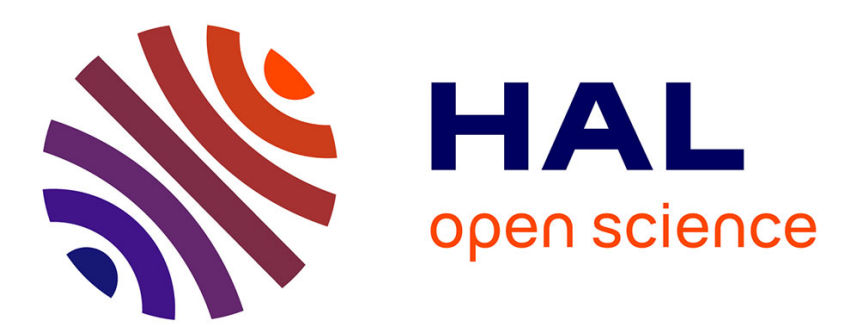

\title{
A Variational Calculus Approach to Wildfire Monitoring Using a Low-Discrepancy Sequence-Based Deployment of Sensors
}

Didier Georges

\section{- To cite this version:}

Didier Georges. A Variational Calculus Approach to Wildfire Monitoring Using a Low-Discrepancy Sequence-Based Deployment of Sensors. CDC 2019 - 58th IEEE Conference on Decision and Control, Dec 2019, Nice, France. hal-02334553

\section{HAL Id: hal-02334553 https://hal.science/hal-02334553}

Submitted on 26 Oct 2019

HAL is a multi-disciplinary open access archive for the deposit and dissemination of scientific research documents, whether they are published or not. The documents may come from teaching and research institutions in France or abroad, or from public or private research centers.
L'archive ouverte pluridisciplinaire HAL, est destinée au dépôt et à la diffusion de documents scientifiques de niveau recherche, publiés ou non, émanant des établissements d'enseignement et de recherche français ou étrangers, des laboratoires publics ou privés. 


\title{
A Variational Calculus Approach to Wildfire Monitoring Using a Low-Discrepancy Sequence-Based Deployment of Sensors
}

\author{
Didier Georges
}

\begin{abstract}
This paper deals with the estimation of the wildfire ignition location by using a variational approach, which, to the best of my knowledge, has never been proposed before. Wildfires are here modeled by using two balance equations for energy and fuel, where the fuel loss due to combustion is defined by the fuel reaction rate. The physical coefficients of the model, together with the initial fuel distribution, are here supposed to be known. The use of a small number of low cost temperature sensors constituting a sensor network distributed on the field according to a low-discrepancy sequence is investigated which provides some promising results for this estimation problem considered to be very difficult in the litterature.
\end{abstract}

\section{INTRODUCTION}

In the context of ever-increasing climate change, the issue of wildfire real-time monitoring is a major concern. The litterature proposes a large number of references devoted to the dynamics of fire propagation. [1] provides an extensive review of physical and quasi-physical models used to represent the dynamics of wildfires. Very sophisticated physical models based on extended irreversible thermodynamics [2] have been proposed. Such models are very complex and very expensive from a numerical simulation point of view, therefore we are not very suitable for solving estimation and data assimilation problems. Quasi-physical models based on the level set method set governed by a Hamilton-Jacobi equation have also been studied [3].

This paper is devoted to the early detection of a fire ignition using a variational approach applied to a wildfire propagation model based on two $2 \mathrm{D}$ coupled partial differential equations. This model offers a good trade-off between complexity and the ability to realistically represent physical phenomena, such as traveling heat waves [4]. A first equation represents the transport and diffusion of temperature due to the fuel combustion reaction, while the second equation represents the dynamics of fuel loss. Ensemble Kalman filters are used in the literature for wildfire estimation applications (see [4] or [5] for instance). As far as I know, this paper is the first attempt to use a variational approach to locate a fire ignition. The here-proposed approach extends the work in [7] to advection-diffusion problems in 2D. Th proposed approach belongs to the class of optimization problems with PDE constraints discussed in [10] and [11].

The paper is now organized as follows: Section 2 presents the modeling of wildfires. The fire ignition estimation ap-

This work is supported by the French National Research Agency in the framework of the Investissements d'Avenir program (ANR-15-IDEX-02).

D. Georges is with Univ. Grenoble Alpes, CNRS, Grenoble INP, GIPSA-lab, 38000 Grenoble, France, didier.georges@grenoble-inp.fr proach is proposed in section 3. Section 4 discusses the numerical method used to solve the optimal estimation problem. Some numerical simulations of a wildfire based on real physical chararacterics together with some preliminary fire ignition estimation results are presented in section 5. Some conclusions and perspectives are given in section 6 . The detailed derivation of the variational calculus approach is given in Appendix.

\section{WILDFIRE MODELING}

In this paper, the model proposed in [4] is used. This model consists in two 2D coupled partial differential equations, which define the energy balance and fuel reaction rate for a wildfire in a ground layer of some given finite small thickness, on a rectangular domain $D=\left[0, L_{x}\right] \times\left[0, L_{y}\right]$ :

$$
\begin{aligned}
\partial_{t} T & =\partial_{x}\left(k \partial_{x} T\right)+\partial_{y}\left(k \partial_{y} T\right)-v_{x} \partial_{x} T-v_{y} \partial_{y} T \\
& +A\left(\operatorname{Sr}(T)-C\left(T-T_{a}\right)\right), \\
\partial_{t} S & =-C_{S} \operatorname{Sr}(T),
\end{aligned}
$$

with Arrhenius reaction rate from physical chemistry

$$
r(T)=\left\{\begin{array}{c}
e^{-B /\left(T-T_{a}\right)}, T>T_{a} \\
0, T \leq T_{a}
\end{array}\right.
$$

and where $T(x, y, t)$ is the distributed temperature in the ground layer, $S(x, y, t)$ is the distributed mass fraction of fuel. $k$ is the coefficient of temperature diffusion. $v=$ $\left(v_{x}(x, y, t), v_{y}(x, y, t)\right)$ defines the velocity field of the air, supposed to known from meteorological data. $A, B, C, C_{S}$ are some physical coefficients. $T_{a}$ is the ambiant temperature. $\partial_{t}$, $\partial_{x}$, and $\partial_{y}$ denote the partial derivatives with respect to time $t$, and spatial coordinates $x$ and $y$, respectively.

Some boundary and initial conditions have also to be defined to ensure the well-posedness of the problem. Neumann's boundary conditions are used in this paper:

$$
\begin{array}{r}
\partial_{x} T(0, y, t)=\partial_{x} T\left(L_{x}, y, t\right)=0, \forall y \in\left[0, L_{y}\right], \\
\partial_{y} T(x, 0, t)=\partial_{y} T\left(x, L_{y}, t\right)=0, \forall x \in\left[0, L_{x}\right], \\
T(x, y, 0)=T_{0}(x, y), S(x, y, 0)=S_{0}(x, y), \forall(x, y) \in D .
\end{array}
$$

The interest of this model lies in the fact it is able to simulate heat travelling waves in a realistic way.

It is also convenient to introduce nondimensional variables and dimensionless coefficients. The following changes of variables

$$
\begin{array}{r}
\tilde{T}=\frac{T-T_{a}}{B}, \tilde{v}=\frac{B v}{A}, \tilde{x}=\frac{x}{k^{1 / 2} B^{1 / 2} A^{-1 / 2}}, \\
\tilde{y}=\frac{y}{k^{1 / 2} B^{1 / 2} A^{-1 / 2}}, \tilde{t}=\frac{A t}{B}
\end{array}
$$


transform (1)-(2) into the nondimensional form

$$
\begin{aligned}
\partial_{\tilde{t}} \tilde{T} & =\partial_{\tilde{x} \tilde{x}} \tilde{T}+\partial_{\tilde{y} \tilde{y}} \tilde{T}-\tilde{v}_{x} \partial_{\tilde{x}} \tilde{T}-\tilde{v}_{y} \partial_{\tilde{y}} \tilde{T} \\
& +\tilde{S} e^{-1 / \tilde{T}}-\lambda \tilde{T}, \\
\partial_{\tilde{t}} \tilde{S} & =-\beta \tilde{S} e^{-1 / \tilde{T}}, \tilde{T}>0,
\end{aligned}
$$

with the following two dimensionless coefficients

$$
\lambda=C B, \beta=\frac{B C_{S}}{A} .
$$

In what follows, the tildes will be removed for the ease of notation.

\section{OPTIMAL ESTIMATION PROBLEM}

The objective of this paper is to explore the feasibility of an early detection and location of fire ignition from a limited number of temperature measurements apart from sensors deployed in the field. A network of low cost temperature sensors is assumed to be deployable in the field. As these sensors can be exposed to very high temperatures during a fire, the risk of sensor dysfunctioning is here taken into account. Here it is assumed that the initial fuel distribution $S(x, y, 0)$ is known from an a priori mapping of the field. Coefficients $\beta$ and $\lambda$ are also assumed to be known from the knowledge of previous wildfire occurences with the same fuel characteristics.

\section{A. Formulation}

The fire ignition estimation will consist in finding the initial distributed temperature in domain $D$. This can be formulated as solving the optimal least-square optimization problem defined for $N_{s}$ sensors and on time interval $\left[0, T_{f}\right]$ by

$$
\begin{array}{r}
\min _{T(x, y, 0) \in D} J\left(T_{0}\right)=\min _{T(x, y, 0) \in D} \frac{1}{2} \sum_{i=1}^{N_{s}} \int_{0}^{T_{f}}\left(y_{i}(t)-y_{i}^{m}(t)\right)^{2} d t \\
+\frac{\alpha}{2} \int_{0}^{L_{x}} \int_{0}^{L_{y}} T(x, y, 0)^{2} d x d y,
\end{array}
$$

subject to

$$
\begin{aligned}
\partial_{t} T & =\partial_{x x} T+\partial_{y y} T-v_{x} \partial_{x} T-v_{y} \partial_{y} T \\
& +S e^{-1 / T}-\lambda T \\
\partial_{t} S & =-\beta S e^{-1 / T}, T>0,
\end{aligned}
$$

where the measurement operator for each sensor $i$ is given by

$$
y_{i}(t)=\psi\left(\bar{y}_{s}^{i}(t)\right) \bar{y}_{s}^{i}(t)
$$

with $\bar{y}_{s}^{i}(t)=\int_{0}^{L_{x}} \int_{0}^{L_{y}} \Delta\left(x-x_{s}^{i}, y-y_{s}^{i}\right) T(x, y, t) d x d y$, and where pair $\left(x_{s}^{i}, y_{s}^{i}\right)$ denotes the spatial coordinates of sensor $i$ in the reference frame. $\Delta$ defines the measurement characteristic of the sensor (this is the Dirac function in case of measurement at a very specific point of the domain). $\Delta$ is here chosen as a Gaussian kernel that provides adjustable local averaging of the temperature. Function $\psi($.$) represents$ the confidence in measurement related to possible sensor dysfunctioning, which is decreasing when the local temperature at the vicinity of the sensor increases, and is here defined by

$$
\psi(y)=\frac{1}{1+e^{K\left(y-T_{f}\right)}}
$$

where $T_{f}$ denotes the threshold temperature at which the confidence value is equal to 0.5 . When $\psi(y)$ is equal to one, measurement $y$ is considered as fully reliable. When $\psi(y)$ is equal to zero, the measurement is not taken into account at all. $K>0$ is used to tune the measurement confidence according to the sensor resistance to fire. In this paper, it is supposed that the sensors are still operating after exposure to the high temperature induced by the fire, that is a rather strong assumption. A true (but more complex) failure model should be considered in practice.

Fig 1 shows an example of $\psi$ for $K=1$ and $T_{f}=1.5$ :

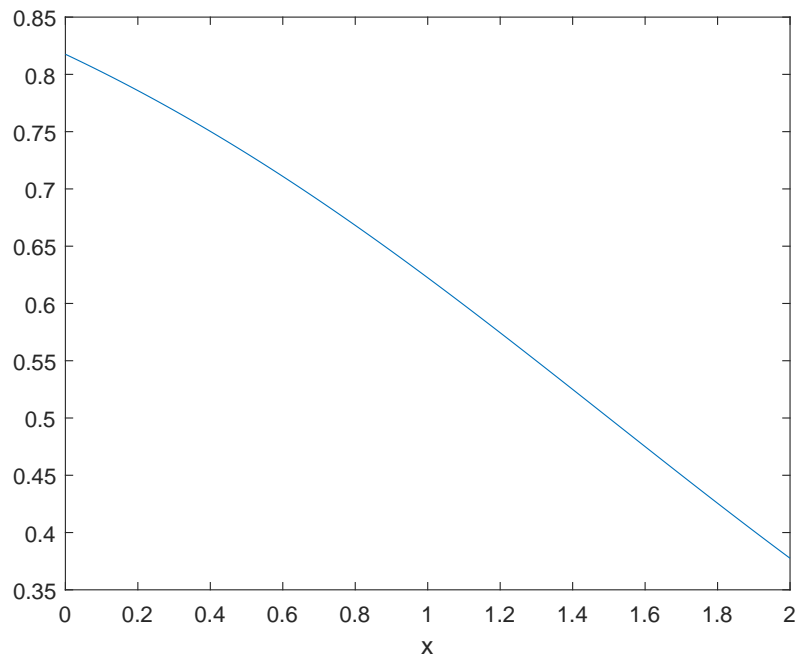

Fig. 1. Function $\psi(y)$.

$y_{i}^{m}(t)$ is the measured data provided by sensor $i$. Finally $\alpha$ represents a regularization coefficient.

\section{B. Low-Discrepancy Sequence Deployment of the Sensors}

The use of low-discrepancy sequences such as the ones proposed by Halton, Sobol, Faure (see [6] for instance) to generate learning sequences is investigated in this paper.

Such sequences have been proposed to solve the problem of optimally choosing M samples $x_{i}$ in a hypercube $C=$ $[0,1]^{n}$ to "minimize holes" in the sense of the best possible approximation of integrals:

$$
\left|\frac{1}{M} \sum_{i=1}^{M} f\left(x_{i}\right)-\int_{C} f(x) d x\right| \leq V(f) \frac{\log (M)^{n}}{M}
$$

$V(f)$ is the variation of $f$ in the sense of Hardy \& Krause.

This approach usually provides better approximation results than other approaches based on random sequences for $n \leq 20$.

Fig. 6 shows the distribution of 25 sensors on a square domain according to Sobol's sequence. 


\section{Necessary Conditions for Optimality}

In order to derive the first-order necessary conditions for optimality fo this problem, a Lagrangian formulation is introduced:

$$
\begin{array}{r}
L\left(T, S, p_{1}, p_{2}\right)=\frac{1}{2} \sum_{i=1}^{N_{s}} \int_{0}^{T_{f}}\left(y_{i}(t)-y_{i}^{m}(t)\right)^{2} d t \\
+\frac{\alpha}{2} \int_{0}^{L_{x}} \int_{0}^{L_{y}} T(x, y, 0)^{2} d x d y \\
+\int_{0}^{T_{f}} \int_{0}^{L_{x}} \int_{0}^{L_{y}} p_{1}(x, y, t)\left[\partial_{t} T-\partial_{x x} T-\partial_{y y} T\right. \\
\left.\quad+v_{x} \partial_{x} T+v_{y} \partial_{y} T-S e^{-1 / T}+\lambda T\right] d x d y d t \\
+\int_{0}^{T_{f}} \int_{0}^{L_{x}} \int_{0}^{L_{y}} p_{2}(x, y, t)\left[\partial_{t} S+\beta S e^{-1 / T}\right] d x d y d t
\end{array}
$$

with boundary and initial conditions

$$
\begin{array}{r}
\partial_{x} T(x, y, t)=\partial_{y} T(x, y, t)=0, \forall(x, y) \in \partial D, \\
T(x, y, 0)=T_{0}(x, y), S(x, y, 0)=S_{0}(x, y), \forall(x, y) \text { in } D,
\end{array}
$$

where $p_{1}(x, y, t)$ and $p_{2}(x, y, t)$ are the so-called Lagrange multipliers also called adjoint variables.

If a solution to this optimization problem exists and provided that this solution has sufficient regularity, then the optimality conditions can be derived as follows:

$$
\begin{array}{r}
\partial_{t} T=\partial_{x x} T+\partial_{y y} T-v_{x} \partial_{x} T-v_{y} \partial_{y} T+\tilde{S} e^{-1 / T}-\lambda T, \\
\partial_{x} T(x, y, t)=\partial_{y} T(x, y, t)=0, \forall(x, y) \in \partial D, \\
\partial_{t} S=-\beta S e^{-1 / T}, \\
S(x, y, 0)=S_{0}(x, y), \forall(x, y) i n D, \\
\sum_{i=1}^{N_{s}} \Delta\left(x-x_{s}^{i}, y-y_{s}^{i}\right)\left[\bar{y}_{s}^{i}-y_{i}^{m}\right] \\
\times\left[\psi\left(\bar{y}_{s}^{i}\right)+\frac{d \psi}{d y}\left(\bar{y}_{s}^{i}\right) \bar{y}_{s}^{i}\right] \\
S \\
-\partial_{t} p_{1}-\partial_{x x} p_{1}-\partial_{y y} p_{1}-p_{1} \frac{T^{2}}{T^{-1 / T}} \\
+\lambda p_{1}+p_{2} \frac{\beta S}{T^{2}} e^{-1 / T}=0, \\
v_{x} p_{1}(0, y, t)+\partial_{x} p_{1}(0, y, t)=0, \\
\partial_{x} p_{1}\left(L_{x}, y, t\right)=0, \forall y \in\left[0, L_{y}\right], \\
v_{y} p_{1}(x, 0, t)+\partial_{y} p_{1}(x, 0, t)=0, \\
v_{x} p_{1}\left(L_{x}, y, t\right)+p_{1}\left(x, L_{y}, t\right)=0, \forall x \in\left[0, L_{x}\right], \\
\forall t \in\left[0, T_{f}\right], \\
v_{y} p_{1}\left(x, L_{y}, t\right)+\partial_{y} \partial_{t} p_{2}-p_{1} e^{-1 / T}+p_{2} \beta e^{-1 / T}=0, \\
p_{1}\left(x, y, T_{f}\right)=p_{2}\left(x, y, T_{f}\right)=0, \forall(x, y) \in D, \forall t \in\left[0, T_{f}\right], \\
\alpha T(x, y, 0)-p_{1}(x, y, 0)=0, \forall(x, y) \in D .
\end{array}
$$

(20)-(23) define the dynamics of the estimated system. (24)(27) represent the adjoint system well defined by boundary and terminal time conditions. (28) is the fact that the gradient of the cost function of optimization problem (11) has to be equal to zero at optimality.

The details of the derivation are given in the appendix.

\section{NUMERICAL METHOD}

In order to solve necessary conditions (20)-(27), many methods, such as the quasi-Newton method [8], can be used. All the available descent methods rely on the computation of the gradient of the cost function.

To compute the gradient of cost function (11) given by (28), the discretization of both $T(x, y, t), S(x, y, t), p_{1}(x, y, t)$, and $p_{2}(x, y, t)$ is performed on a space-time grid $\left(x_{i}, y_{j}, t_{l}\right)$. The procedure is the following:

Suppose that an estimate of initial temperature distribution $T\left(x_{i}, y_{j}, 0\right)$ on spatial grid $\left(x_{i}, y_{j}\right)$, obtained at a given iteration:

- Solve PDEs (20) and (22) with conditions (21) and (23) by using finite-difference approximations of the spatial partial differential operators (using the so-called method of lines) on spatial grid $\left(x_{i}, y_{j}\right)$, with a time integration performed forward in time on the time grid $\left(t_{l}\right)$.

- Solve adjoint PDEs (24) and (26) with conditions (25) and (27) by using the same finite-difference approximations on spatial grid $\left(x_{i}, y_{j}\right)$, with a time integration performed backward in time, since only terminal time conditions given by (27) are available.

- Compute the gradient $\alpha T\left(x_{i}, y_{j}, 0\right)-p_{1}\left(x_{i}, y_{j}, 0\right)$ on spatial grid $\left(x_{i}, y_{j}\right)$.

Here a simple gradient descent method with a randomly chosen initial estimate of the temperature has been successfully used to get the results presented in what follows.

\section{NUMERICAL RESULTS}

Throughout this section, the physical coefficients values were the ones given in [4]: $A=1.8793 \times 10^{2}(\mathrm{~K} / \mathrm{s})$ $B=5.5849 \times 10^{4}(K), \quad C=4.8372 \times 10^{-5}(1 / K), \quad C_{S}=$ $1.625^{-1}(1 / s), k=2.136 \times 10^{-1}\left(\mathrm{~m}^{2} / s K^{3}\right)$, ambient temperature $T_{a}=300(K)$, and ignition temperature $T_{c}=1200(K)$. The wind velocities are assumed to be equal to zero. All the simulations were performed using nondimensionnal representation (8)-(9).

\section{A. Simulation of the Wildfire Model}

All the simulations were carried out in domain $D=$ $\left[0, L_{x}\right] \times\left[0, L_{y}\right]$, with $L_{x}=L_{y}=650$, which correspond to a square of 500 meters on each side, that may constitute an elementary cell of a larger field to be monitored. The fire model was implemented by central finite differences in space. The spatial grid size was $80 \times 80$. The initial temperature $T_{0}(x, y)$ on the domain was given by a gaussian distribution of the form $T_{0}(x, y)=T_{c} e^{-\frac{\left\|(x, y)-\left(x_{i}, y_{i}\right)\right\|^{2}}{2 \sigma^{2}}}+T_{a}$, where $\left(x_{i}=\right.$ $\left.400, y_{i}=350\right)$ denote the coordinates of the ignition location in the reference frame. The distribution of fuel is uniform except for a fuel break (a road or a river) which was introduced as depicted in Fig. 2. Fig. 3 shows the initial gaussian distribution of the temperature. Fig. 4 and Fig. 5 present the fuel consumption and the temperature distribution at $t=670$. 


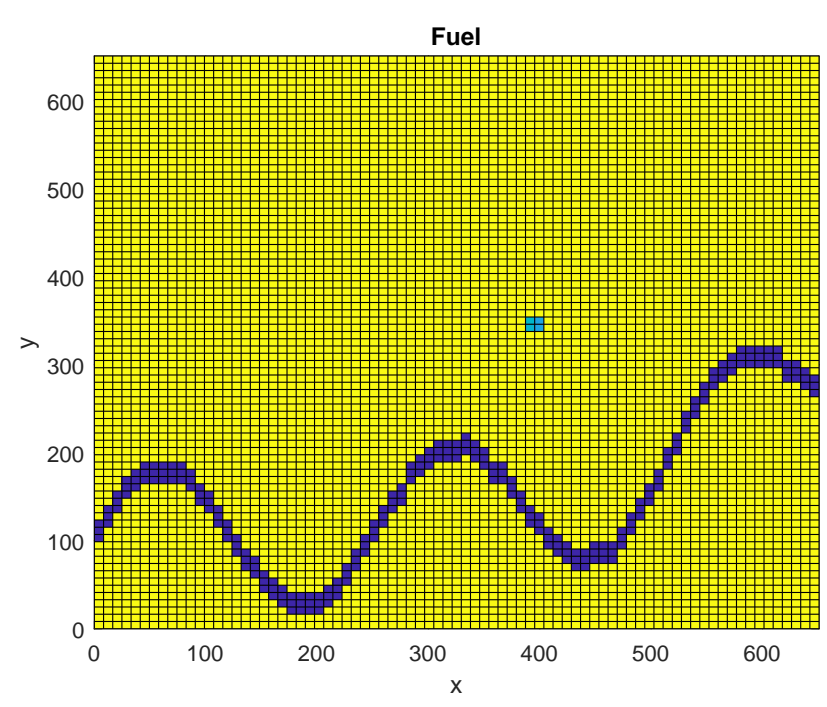

Fig. 2. Fire ignition location with a fuel break.

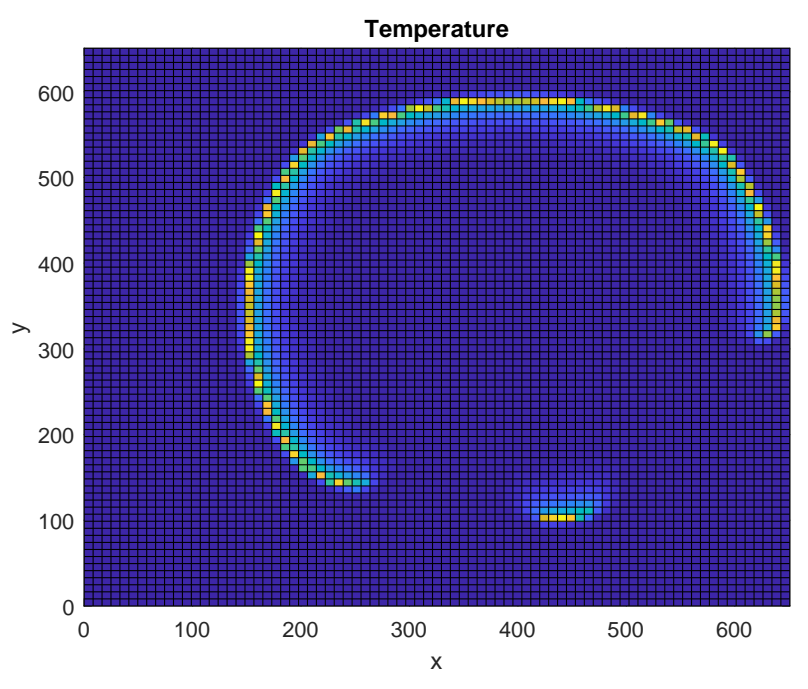

Fig. 5. Temperature distribution at $t=670$.

\section{B. Estimation of Fire Ignition}

In this section, the use of 25 ground temperature sensors deployed according to a Sobol's low discrepancy sequence is investigated. Fig. 6 shows the corresponding location of the sensors in the domain.

Estimation horizon $T_{f}$ was equal to 670 (corresponding to $1990 s$ ). Regularization coefficient $\alpha$ was equal to 1 . Confidence function $\phi$ was defined with $K=1$ and failure temperature $T_{f}=1.5$ (corresponding to $1800 \mathrm{~K}$ ). The gradient descent algorithm was initialized using a randomly distributed temperature. Fig. 7 shows the estimated initial temperature distribution of the real initial temperature distribution given in Fig.3. The algorithm provides a fairly accurate estimate of the location with relative quadratic error less than. Fig. 8 shows the estimated temperature distribution at $t=670$ after the fire ignition. A quite accurate estimate of the heat wave can be obtained (compare with the reference solution given in Fig. 5). The average CPU time needed to solve the estimation problem is $50 \mathrm{~s}$ on a computer equipped with a Intel Core I7. The choice of the regularization coefficient appears to be quite critical to get good results. A too high value of this coefficient leads to the algorithm being stuck at an inappropriate local minimum, while a too small value prevents the gradient method to properly converge. The results also are quite sensitive to the algorithm initialization what seems confirm the ill-posedness of this fire estimation problem.

\section{CONCLUSIONS AND FUTURE WORKS}

In this paper a preliminary investigation of the use of an infinite-dimensional optimal estimation approach using low cost temperature sensors deployed according a lowdiscrepancy sequence has been carried out. The simulations results demonstrate promising results of the proposed approach. Further work needs to be done to address theoretical issues such as regularity of the solution and differentiability of the objective function, and to analyze whether the

Fig. 4. Fuel consumption (in dark blue) at $t=670$. 


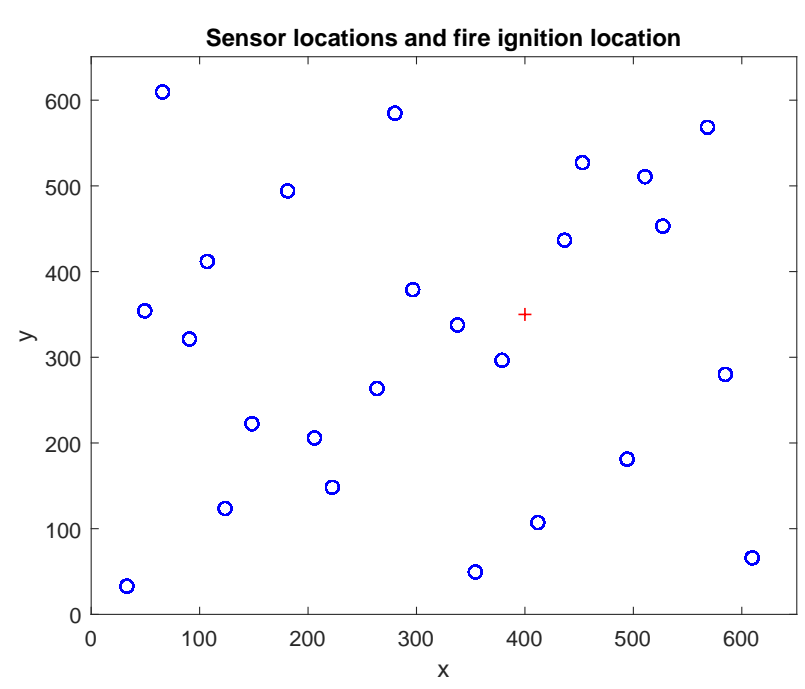

Fig. 6. 25-Sensor location and fire ignition location (red cross).

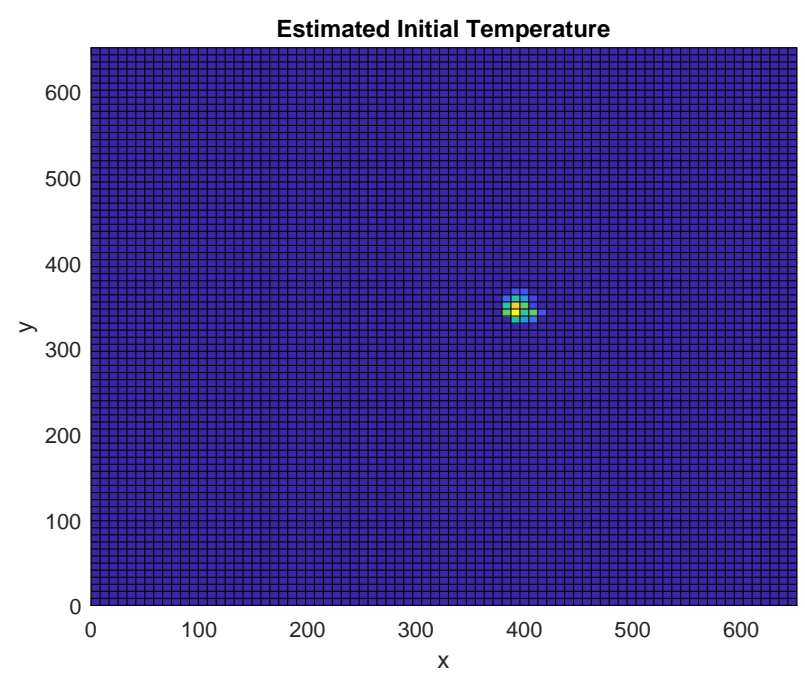

Fig. 7. Estimated fire ignition.

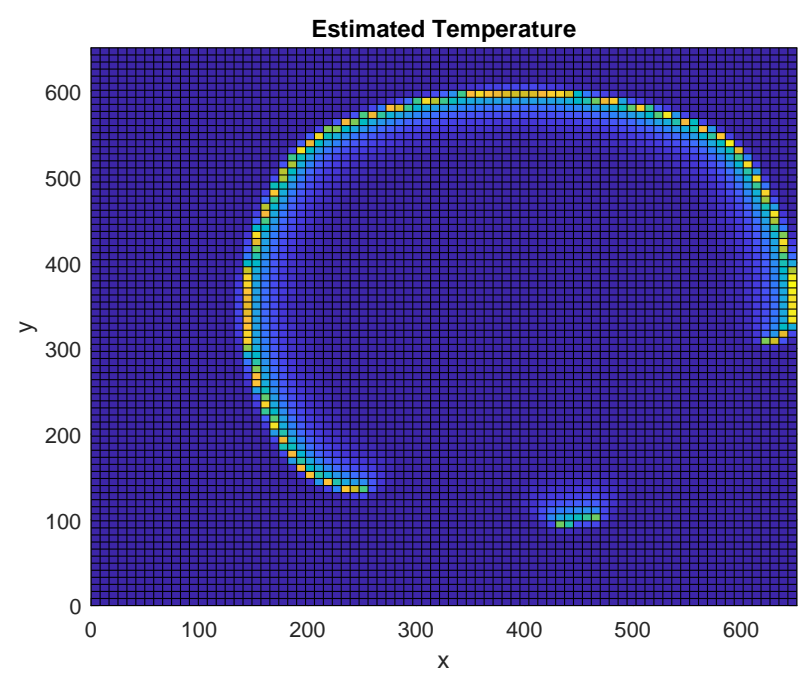

Fig. 8. Estimated temperature distribution at $t=670$. heuristic placement of sensors according to a low discrepancy sequence has real optimality properties in terms of observability or whether it is better to use a optimal sensor location approach similar to the one proposed in [9] for instance.

\section{APPENDIX}

In order to derive necessary conditions for optimality (20)(28), and according to the fundamental theorem of Calculus of Variations, the Gateaux derivative of cost function of problem (11) with respect to initial temperature $T_{0}$ in direction $\delta T_{0}$ has to be equal to zero

$$
\lim _{\varepsilon \rightarrow 0} \frac{J\left(T_{0}+\varepsilon \delta T_{0}\right)-J\left(T_{0}\right)}{\varepsilon}=0,
$$

or, equivalently, the derivatives of Lagrangian (17) with respect to $T$ and $S$ in directions $\delta T$ and $\delta S$, respectively, have to be equal to zero. The computation of these directional derivatives will lead to the derivation of necessary conditions (20)-(28)

Five integrations by parts of the Lagrangian function have to be performed (one with respect to time, and two consecutive ones with respect to each state coordinate $x$ and $y$ ), leading, after easy but quite lengthy calculations, to the following expression of the Lagrangian:

$$
\begin{array}{r}
L\left(T, S, p_{1}, p_{2}\right)=\frac{1}{2} \sum_{i=1}^{N_{s}} \int_{0}^{T_{f}}\left(y_{i}(t)-y_{i}^{m}(t)\right)^{2} d t \\
+\frac{\alpha}{2} \int_{0}^{L_{x}} \int_{0}^{L_{y}} T(x, y, 0)^{2} d x d y \\
+\int_{0}^{L_{x}} \int_{0}^{L_{y}}\left[p_{1}\left(x, y, T_{f}\right) T\left(x, y, T_{f}\right)\right. \\
\left.\quad-p_{1}(x, y, 0) T(x, y, 0)\right] d x d y
\end{array}
$$

$$
\begin{array}{r}
+\int_{0}^{T_{f}} \int_{0}^{L_{y}}\left[\left(v_{x} p_{1}\left(L_{x}, y, t\right)+\partial_{x} p_{1}\left(L_{x}, y, t\right)\right) T\left(L_{x}, y, t\right)\right. \\
\left.-\left(v_{x} p_{1}(0, y, t)+\partial_{x} p_{1}(0, y, t)\right) T(0, y, t)\right] d y d t \\
+\int_{0}^{T_{f}} \int_{0}^{L_{x}}\left[\left(v_{y} p_{1}\left(x, L_{y}, t\right)+\partial_{y} p_{1}\left(x, L_{y}, t\right)\right) T\left(x, L_{y}, t\right)\right. \\
\left.-\left(v_{y} p_{1}(x, 0, t)+\partial_{y} p_{1}(x, 0, t)\right) T(x, 0, t)\right] d x d t \\
+\int_{0}^{T_{f}} \int_{0}^{L_{x}} \int_{0}^{L_{y}}\left[\left(-\partial_{t} p_{1}-v_{x} \partial_{x} p_{1}-v_{y} \partial_{y} p_{1}\right.\right. \\
\left.\left.-\partial_{x x} p_{1}-\partial_{y y} p_{1}\right) T-p_{1} S e^{-1 / T}+p_{1} \lambda T\right] d x d y d t \\
+\int_{0}^{L_{x}} \int_{0}^{L_{y}}\left[p_{2}\left(x, y, T_{f}\right) S\left(x, y, T_{f}\right)\right. \\
\left.-p_{2}(x, y, 0) T(x, y, 0)\right] d x d y \\
+\int_{0}^{T_{f}} \int_{0}^{L_{x}} \int_{0}^{L_{y}}\left[-\partial_{t} p_{2} S+p_{2} \beta S e^{-1 / T}\right] d x d y d t
\end{array}
$$

The directional derivatives of Lagrangian (30) with respect to $T$ and $S$ in directions $\delta T(x, y, t), \delta T(x, y, 0), \delta T\left(x, y, T_{f}\right)$, $\delta T(0, y, t), \delta T\left(L_{x}, y, t\right), \delta T(x, 0, t), \delta T\left(x, L_{y}, t\right), \delta S(x, y, t)$, and $\delta S\left(x, y, T_{f}\right)$ are now computed and set equal to zero: 
- Derivative in direction $\delta T(x, y, t)$ :

$$
\begin{array}{r}
\sum_{i=1}^{N_{s}} \int_{0}^{T_{f}} \int_{0}^{L_{x}} \int_{0}^{L_{y}} \Delta\left(x-x_{s}^{i}, y-y_{s}^{i}\right)\left[\bar{y}_{s}^{i}-y_{i}^{m}\right] \\
\times\left[\psi\left(\bar{y}_{s}^{i}\right)+\frac{d \psi}{d y}\left(\bar{y}_{s}^{i}\right) \bar{y}_{s}^{i}\right] \delta T d x d y d t \\
+\int_{0}^{T_{f}} \int_{0}^{L_{x}} \int_{0}^{L_{y}}\left[\left(-\partial_{t} p_{1}-v_{x} \partial_{x} p_{1}-v_{y} \partial_{y} p_{1}\right.\right. \\
\left.-\partial_{x x} p_{1}-\partial_{y y} p_{1}\right) \delta T-p_{1}\left(\delta S e^{-1 / T}+\frac{S}{T^{2}} e^{-1 / T} \delta T\right) \\
\left.+p_{1} \lambda \delta T\right] d x d y d t \\
+\int_{0}^{T_{f}} \int_{0}^{L_{x}} \int_{0}^{L_{y}} p_{2} \frac{\beta S}{T^{2}} e^{-1 / T} \delta T d x d y d t=0, \\
\forall \delta T(x, y, t),
\end{array}
$$

which leads to first adjoint PDE (24) by collecting all the terms under the integrals.

- Derivative in direction $\delta T(x, y, 0)$ :

$$
\begin{array}{r}
\int_{0}^{L_{x}} \int_{0}^{L_{y}}\left[\alpha T(x, y, t)-p_{1}(x, y, 0] \delta T(x, y, 0) d x d y=0,\right. \\
\forall \delta T(x, y, 0),
\end{array}
$$

which leads to condition (28) and gives the expression of the gradient of the cost function.

- Derivative in direction $\delta T\left(x, y, T_{f}\right)$ :

$$
\begin{array}{r}
\int_{0}^{L_{x}} \int_{0}^{L_{y}} p_{1}\left(x, y, T_{f}\right) \delta T\left(x, y, T_{f}\right) d x d y=0, \\
\forall \delta T\left(x, y, T_{f}\right),
\end{array}
$$

which leads to terminal time condition $p_{1}\left(x, y, T_{f}\right)=0$ in (27).

- Derivative in direction $\delta T(0, y, t)$ :

$$
\begin{gathered}
-\int_{0}^{T_{f}} \int_{0}^{L_{y}}\left(v_{x} p_{1}(0, y, t)+\partial_{x} p_{1}(0, y, t)\right) \\
\quad \times \delta T(0, y, t) d y d t=0, \forall \delta T(0, y, t),
\end{gathered}
$$

leading to adjoint boundary condition $v_{x} p_{1}(0, y, t)+$ $\partial_{x} p_{1}(0, y, t)=0$ in $(25)$.

- Derivative in direction $\delta T\left(L_{x}, y, t\right)$ :

$$
\begin{aligned}
& \int_{0}^{T_{f}} \int_{0}^{L_{y}}\left(v_{x} p_{1}\left(L_{x}, y, t\right)+\partial_{x} p_{1}\left(L_{x}, y, t\right)\right) \\
& \quad \times \delta T\left(L_{x}, y, t\right) d y d t=0, \forall \delta T\left(L_{x}, y, t\right),
\end{aligned}
$$

leading to adjoint boundary condition $v_{x} p_{1}\left(L_{x}, y, t\right)+$ $\partial_{x} p_{1}\left(L_{x}, y, t\right)=0$ in $(25)$.

- Derivative in direction $\delta T(x, 0, t)$ :

$$
\begin{array}{r}
-\int_{0}^{T_{f}} \int_{0}^{L_{x}}\left(v_{y} p_{1}(x, 0, t)+\partial_{y} p_{1}(x, 0, t)\right) \\
\quad \times \delta T(x, 0, t) d x d t=0, \forall \delta T(x, 0, t),
\end{array}
$$

leading to adjoint boundary condition $v_{y} p_{1}(x, 0, t)+$ $\partial_{y} p_{1}(x, 0, t)=0$ in $(25)$.

- Derivative in direction $\delta T\left(x, L_{y}, t\right)$ :

$$
\begin{array}{r}
\int_{0}^{T_{f}} \int_{0}^{L_{x}}\left(v_{y} p_{1}\left(x, L_{y}, t\right)+\partial_{y} p_{1}\left(x, L_{y}, t\right)\right) \\
\quad \times \delta T\left(x, L_{y}, t\right) d x d t=0, \forall \delta T\left(x, L_{y}, t\right),
\end{array}
$$

leading to adjoint boundary condition $v_{y} p_{1}\left(x, L_{y}, t\right)+$ $\partial_{y} p_{1}\left(x, L_{y}, t\right)=0$ in $(25)$.

- Derivative in direction $\delta S(x, y, t)$ :

$$
\begin{array}{r}
-\int_{0}^{T_{f}} \int_{0}^{L_{x}} \int_{0}^{L_{y}} p_{1} e^{-1 / T} \delta S d x d y d t \\
+\int_{0}^{T_{f}} \int_{0}^{L_{x}} \int_{0}^{L_{y}}\left[-\partial_{t} p_{2}+p_{2} \beta e^{-1 / T}\right] \delta S d x d y d t=0, \\
\forall \delta S(x, y, t),
\end{array}
$$

leading to second adjoint PDE (26), after collecting the terms under the integrals.

- Derivative in direction $\delta T\left(x, y, T_{f}\right)$ :

$$
\begin{array}{r}
\int_{0}^{L_{x}} \int_{0}^{L_{y}} p_{2}\left(x, y, T_{f}\right) \delta S\left(x, y, T_{f}\right) d x d y=0, \\
\forall \delta S\left(x, y, T_{f}\right),
\end{array}
$$

which leads to terminal time condition $p_{2}\left(x, y, T_{f}\right)=0$ in (27).

Finally system PDEs (20) and (22) governing the wildfire dynamics with their boundary and initial conditions (21) and (23) complete the set of first-order necessary conditions for optimality. The initial distribution of temperature $T(x, y, 0)$, which is the main optimization variable, is obtained from the stationary condition of the gradient of the cost function (28).

\section{REFERENCES}

[1] A. L. Sullivan, Wildland surface fire spread modelling, 1990-2007. 1: Physical and quasi-physical models, International Journal of Wildland Fire, 2009, 18, 349-368.

[2] O. Sero-Guillaume, and J. Margerit, Modelling forest fires. Part I: A complete set of equations derived by extended irreversible thermodynamics, International Journal of Heat and Mass Transfer, 2002 45(8):1705-1722.

[3] M. Feckan, and J. Pacuta, Existence of solution of a forest fire spread mode, Applied Mathematics Letters, 83 (2018) 227-231.

[4] Jan Mandel, L. S. Bennethuma, J. D. Beezley, J. L. Coen, C. C. Douglas, M. Kima, and A. Vodacek, A wildland fire model with data assimilation, Mathematics and Computers in Simulation, 79 (2008) 584-606.

[5] T. Srivas, T. Artes, R. A. de Callafon, and I. Altintas, Wildfire Spread Prediction and Assimilation for FARSITE using Ensemble Kalman Filtering, Procedia Computer Science Volume 80, 2016, Pages 897-908.

[6] H. Niederreiter, Low-Discrepancy and Low-Dispersion Sequences, Journal of Number Theory, 30: 51-70, 1988.

[7] V. T. Nguyen, D. Georges, and G. Besançon, "State and parameter estimation in 1-D hyperbolic PDEs based on an adjoint method", Automatica, Volume 67, May 2016, Pages 185-191.

[8] J. F. Bonnans, J. Ch. Gilbert, C. Lemaréchal, and C. A. Sagastizábal, Numerical optimization, theoretical and numerical aspects, Second edition, Springer, 2006. ISBN 978-3-540-35445-1.

[9] D. Georges, "Optimal Sensor Location and Mobile Sensor Crowd Modeling for Environmental Monitoring”, IFAC-PapersOnLine, Proceeding of the IFAC World Congress, Toulouse, France, Volume 50 Issue 1, July 2017, Pages 7076-7081.

[10] F. Troltzsch, Optimal Control of Partial Differential Equations: Theory, Methods and Applications, American Mathematical Society, 2010.

[11] M. Hinze, R. Pinnau, M. Ulbrich, and S. Ulbrich, Optimization with PDE Constraints, Mathematical Modelling: Theory and Applications, Springer, 2009. 